\title{
Effects of Credibility Indicators on Social Media News Sharing Intent
}

\author{
Waheeb Yaqub ${ }^{\dagger, \sharp} \quad$ Otari Kakhidze $^{\dagger} \quad$ Morgan L. Brockman ${ }^{\ddagger} \quad$ Nasir Memon $^{\S} \quad$ Sameer Patil $^{\ddagger}, \S$ \\ Center for Cybersecurity, New York University, Abu Dhabi, United Arab Emirates \\ \#School of Information Technologies, The University of Sydney, Sydney, Australia \\ $\S$ Tandon School of Engineering, New York University, USA \\ *Luddy School of Informatics, Computing, and Engineering. Indiana University Bloomington, USA \\ wy7@nyu.edu, otto@nyu.edu, morbrock@iu.edu, memon@nyu.edu,patil@indiana.edu
}

\begin{abstract}
In recent years, social media services have been leveraged to spread fake news stories. Helping people spot fake stories by marking them with credibility indicators could dissuade them from sharing such stories, thus reducing their amplification. We carried out an online study $(\mathrm{N}=1,512)$ to explore the impact of four types of credibility indicators on people's intent to share news headlines with their friends on social media. We confirmed that credibility indicators can indeed decrease the propensity to share fake news. However, the impact of the indicators varied, with fact checking services being the most effective. We further found notable differences in responses to the indicators based on demographic and personal characteristics and social media usage frequency. Our findings have important implications for curbing the spread of misinformation via social media platforms.
\end{abstract}

\section{Author Keywords}

Fake news; Misinformation; Disinformation; News headlines; News sharing; Fact-check indicators; Social media; Facebook

\section{ccs Concepts}

-Security and privacy $\rightarrow$ Social aspects of security and privacy; $\cdot$ Human-centered computing $\rightarrow$ Social content sharing; Empirical studies in collaborative and social computing;

\section{INTRODUCTION}

In the aftermath of the Brexit referendum in the United Kingdom and the 2016 Presidential elections in the United States, "Fake News" has come to the forefront as a leading societal and cybersecurity issue. At a high level, the issue involves online misinformation and disinformation (intentional generation of inaccurate or manipulated information) as well as artificial or malicious amplification and spreading of such

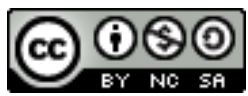

This work is licensed under a Creative Commons Attribution- NonCommercial-

ShareAlike International 4.0 License.

CHI '20, April 25-30, 2020, Honolulu, HI, USA.

(C) 2020 Copyright is held by the owner/author(s). ()ㅇㅇ(-)

ACM ISBN 978-1-4503-6708-0/20/04.

http://dx.doi.org/10.1145/3313831.3376213 content (e.g., via inauthentic accounts or bots) [67]. Such "weaponization of information" [36], especially on critical sociopolitical matters [6], has served to exploit and exacerbate polarization among the public [3].

While misinformation can take many forms, passing factually incorrect information in a form that mimics the characteristics of news stories is a tactic used heavily by malicious or opportunistic actors looking for sociopolitical and/or financial gains from the spread of such stories. To that end, social media services, such as Facebook, have been successfully exploited for amplifying the reach of such "news" stories because of the wide reach of the platforms and their role as a main source of news for many of their users. In fact, people's engagement with fake news stories on Facebook has been shown to be greater than that with legitimate news from mainstream media sources [57], leading to fears of social media platform turning into "weaponized propaganda machines" [49].

Observing that "falsehood diffused significantly farther, faster, deeper, and more broadly than the truth in all categories of information" [65], industry and academia have recognized the urgent need for multidisciplinary efforts to combat the spread of misinformation via online platforms $[18,21,29,55,70]$. The multi-faceted and sociotechnical nature of the issues involved requires correspondingly multi-faceted and sociotechnical approaches to address the problem. For instance, techniques based on detecting unusual social network traffic or bot activity can be applied at scale but may not be effective when misinformation spreads organically via real human activity on the platform. On the other hand, employing moderators to check and approve every shared story provides high reliability but at a slow pace and small scale. An approach that could potentially complement such techniques, by overcoming some of their shortcomings, involves helping users distinguish fake content from factual stories so that they will be less inclined to share misinformation on online platforms, thus helping curb the spread of such content.

One of the ways of achieving the goal of informing users is via user interface (UI) elements, such as icons and text, that provide cues regarding the credibility of a story [34]. However, there has not yet been a systematic investigation of whether the inclusion of such credibility indicators can influence users 
and reduce their sharing of falsehoods. To fill this gap, we formulated the following research questions:

RQ1: Do credibility indicators presented with a headline lead social media users to reduce their intent for sharing fake news? RQ2: Do different types of credibility indicators exhibit different levels of influence on people's sharing intent?

RQ3: Is the influence of credibility indicators impacted by demographic and contextual factors, such as age, gender, political affiliation, frequency of social media use, etc.?

We hypothesized the answer to each of the above questions to be positive. However, we did not hypothesize how the indicators would compare with each other (RQ2) nor the direction of the impact of demographics (RQ3). To verify the corresponding three hypotheses, we conducted an online study in which participants indicated sharing intent for a mixture of true and non-true news stories based on their headlines. In the treatment conditions, the headline of each non-true story was accompanied by a credibility indicator.

Our findings confirm that credibility indicators are indeed effective in reducing the intent to share false content without affecting the intent to share legitimate news stories. However, the extent of the impact varies based on indicator type, political affiliation, age, gender, and social media usage frequency. By demonstrating the utility of credibility indicators and uncovering important factors that impact their effectiveness for curbing the intent to share fake news, we contribute to the important challenge of combating online misinformation. The UI based solution we investigated has the potential to complement automated approaches, thus forming an important piece within the ecosystem of measures to detect and combat the spread of non-legitimate and malicious content.

\section{RELATED WORK}

Although information sharing has been studied since the 1960s, online misinformation is a relatively new topic and has drawn the attention of scholars from multiple disciplines.

\section{Technical Measures}

One of the key technical strategies to combat misinformation is to detect it via automated techniques [59], such as monitoring fact databases [17], measuring platform activity [48], etc., and subsequently apply supervised learning algorithms for classifying or extracting credibility features $[14,20]$. There is a growing body of literature on detecting misleading content circulated in the digital sphere regardless of semantic form or distribution platform [27]. Similar efforts explore ways to reduce the amplification of fake news by preventive as well as reactive tactics [28]. Most of this research does not pay much attention to the human element (i.e., the motivations and behaviors of users when generating, consuming, and spreading misinformation). As Fernandez and Alani [11] remarked: "Common misinformation management strategies (a) do not go beyond the generation of facts and the early detection of malicious accounts, and (b) tend to focus on the technical and not on the human aspects of the problem."

\section{Regulatory Approaches}

There is a call for legal and regulatory approaches that force relevant stakeholders to curb the spread of misinformation.
Results of a World Economic Forum study indicate that governance based solutions tend to be retrospective and deal with provocative content after "digital wildfires" have already spread and caused damage [66]. Moreover, such solutions typically target individual actors and lack broad coverage of the multiple parties involved in rapid large-scale spread of misinformation [66].

Recently, researchers have argued for changing the regulatory classification of fake news content, especially in the political context. Some have called for considering elections as Critical National Infrastructure [53] while others have advocated elevating cybersecurity debates to the highest levels of government in order to devise effective policy measures against large-scale manipulation of the electorate via massive propagation of misinformation [10], especially via bots $[54,60]$.

\section{User Behavior}

Our study included three interrelated concepts pertaining to user behavior: confirmation bias, perceived source credibility, and desire for socializing.

Ma et al. [32] used structural equation modeling to apply Roger's [51] Diffusion of Innovations (DOI) theory to online news sharing practices. DOI describes the process by which innovation spreads within a social network. In Ma et al.'s [32] study, the decision to share online news substituted the action of an individual engaging in activities that lead to the choice of adopting or rejecting an innovation. Specifically, Ma et al. [32] categorized an individual's personal influence, diffusion networks, and the news stories themselves as factors that influence news sharing. They found that news sharing was driven by individual influences and network tie strengths, with no significant influence of source credibility. The same authors conducted another study examining news sharing intentions through the lens of uses and gratifications (U\&G) theory [25] and found that news sharing on social media platforms is positively influenced by people's desire to seek the gratifications associated with information, social status, and social interactions [30]. While intentions to share were not directly related to the semantic content of the news, prior experience with social media was a significant determinant.

Instead of circulation patterns, Horne and Adali [19] examined the text content of false news, concluding that fake content is often more similar to satire than real news and implying that fake content aims persuade through the availability heuristic rather than strength of argument: "overall title structure and the use of proper nouns in titles are very significant in differentiating fake from real [...] fake news is targeted for audiences who are not likely to read beyond titles and is aimed at creating mental associations between entities and claims."

Kahan et al. [24] found that polarization regarding climate change was greater among people with higher science literacy and numerical ability than those who scored lower on these measures, suggesting that those with higher reasoning capabilities used these abilities to solidify their entrenched beliefs. However, Pennycook and Rand [46] suggest that susceptibility to partisan fake news might be better explained by an absence of reasoning than by biased reasoning per se, especially for 
highly disputed associations between beliefs and reasoning capacities [44]. Therefore, Pennycook and Rand [46] argue that "cultivating or promoting our reasoning abilities should be part of the solution to the kinds of partisan misinformation that circulate on social media."

\section{Sharing Trends}

Although the impact of misinformation has not been widely examined through the lens of user behavior, there are a few studies that report collective user practices. For instance, Gabielkov et al. [15] reported that 59\% of the people in their sample admitted forwarding links to their friends on social media without having read the linked article. Relatedly, a Pew Research Center study found that almost a quarter of the respondents had shared fake political content on online platforms despite claiming general confidence in their ability to identify fake news [4]. An experimental study carried out after the 2017 Italian Elections revealed the role of confirmation bias on sharing decisions; confirmatory information was accepted even when it contained obviously false claims while disagreeable information was ignored and increased group polarization [64].

A large body of research that examines people's sharing decisions is dedicated to studying the reasons underlying the sharing choices rather than inspecting the content that is being shared [35]. The outcomes of such research efforts may inform the algorithmic selection of information sources to limit people's social media information exposure to a significantly narrower spectrum of sources and discourage the consumption of non-legitimate information found via Web search.

\section{Community Influences}

People fail to draw a sharp boundary between the knowledge they possess themselves and the knowledge they access via their community. The major motivation for leveraging community knowledge is providing a fair and effective division of cognitive labor. Such epistemic dependence is key for believing the unknown [16]. In the context of fake news, epistemic dependence emphasizes the role of community knowledge as a structural explanation for the cognitive process that supports amplification of the news. Sloman and Rabb [58] found that "knowing that experts understand a phenomenon gives individuals the sense that they understand it better themselves, but only if they believe they have access to the experts' explanation." Thus, people largely rely on the expertise of other people in making an epistemic judgment regarding whether to believe in a statement. It has been argued that judgments on divisive political issues follow the same pattern; when making political judgments, people rely on the knowledge of others indistinguishably from their own [12].

\section{Perceptions of Credibility}

Oeldorf-Hirsch et al. [39] found that flagging social media memes as disputed did not have significant effects on meme credibility, likelihood to share the meme, or likelihood to seek more information on the topic. Allcott et al. [1] showed people the most-shared false news stories from both sides of the political spectrum during the 2016 U.S. presidential campaign and asked whether they would share the story; results of the study showed that people were much more likely to believe stories that aligned with their political preferences, especially if their social networks exhibited ideological segregation. Others have found similar relationships between ideological segregation and sharing rate on Twitter [33]. Ribeiro et al. [50] investigated the effects of such political polarization on what Twitter users perceive and label as misinformation. The study found that perceptions of fake news on Twitter were highly polarized based on the degree of bias of the users who react to fake-newsrelated URLs and the differences between URL domains that tend to be associated with Democrats or Republicans. Further, Jakesch et al. [22] found that the level of trust in a news story depends mainly on the alignment between political affiliation and the claims in the news article, with the source of the news having no significant influence.

\section{Credibility Indicators}

Nyhan and Reifler [38] documented several instances of a "backfire effect" in which offering fact based corrections increased people's misperceptions, i.e., presenting people with facts strengthened their original inaccurate beliefs. In contrast, more recent studies have shown people heeding factual information even when it challenged their ideological commitments [69]. Relatedly, Nyhan et al. [37] and Pennycook et al. [43] have investigated the effect of credibility indicators concluding that "warnings do lead to a modest reduction in perceived accuracy of fake news relative to a control condition" [43]. Pennycook et al. [43] went a step further and demonstrated what they term as the Implied Truth Effect "the presence of warnings caused untagged stories to be seen as more accurate than in the control." To shed further light on these matters, our study explored several credibility indicators that capture the above aspects such as community influences and technical measures.

\section{METHOD}

We used the various influences uncovered by the literature related to online misinformation discussed in the Related Work section to design a study that addressed the research questions outlined in the Introduction.

\section{Study Design and Flow}

After reading study information and consenting to participate earnestly, each participant was presented with 12 headlines of news articles one after another in random order. The 12 presented headlines were chosen dynamically from a larger set of 72 headlines selected for the study, with 2 well-known and 2 obscure headlines chosen at random within each of three types of headlines: True, False, and Satire. The display characteristics of the headlines, such as font type, font size, etc., were the same for all presented headlines to eliminate the impact of these variations on study results ${ }^{1}$.

Participants in the treatment conditions were shown a red credibility indicator below False or Satire headlines (see Figure 1), but not for those in the True category. Those in the control condition received no indicators. The study consisted of four treatment conditions, each corresponding to one of the four types of credibility indicators we tested:

\footnotetext{
${ }^{1}$ We preserved the capitalization used by the source.
} 
1. Fact Checkers: Multiple fact-checking journalists dispute the credibility of this news.

2. News Media: Major news outlets dispute the credibility of this news.

3. Public: A majority of Americans disputes the credibility of this news.

4. Artificial Intelligence (AI): Computer algorithms using Artificial Intelligence (AI) techniques dispute the credibility of this news.

We chose the four credibility indicators based on a number of considerations. The Fact Checkers indicator is derived from similar mechanisms in the literature and in practical systems, such as Google News and Facebook. However, people do not necessarily judge the credibility of a piece of news based simply on fact checkers. Moreover, some individuals may not trust fact checking sites. Further, a given piece of news may not have been examined by fact checkers. Therefore, we included additional indicators based on other mechanisms people might use for judging the credibility of information encountered online. For instance, professional journalists employed by reputed major news outlets are expected to be trained in information verification and to employ it in their reporting $[8,41,45]$. However, these human based approaches are slow and do not scale. AI based techniques, such as machine learning, are increasingly applied to deal with scale. In fact, several techniques that attempt to detect fake news via automated means are based on AI [7, 31, 42, 47, 52, 56]. Therefore, we included an indicator connected to AI. In addition to these independent sources of verification, it is well-known that social influences affect people's content judgments. In fact, social influence is itself a major factor behind the spread of fake news via social media. To leverage social connections, online social platforms are investigating the potential of crowdsourcing to reduce the spread of fake news $[9,26,40,45,62]$. Therefore, we included a credibility indicator that reflected the majority opinion of the population akin to polls used frequently in news reporting. Further, the Public indicator leverages the fact that expressions of solidarity with "fellow Americans" are common in the United States.

The indicators can be mapped to four typical information sources people tend to consult: experts (Fact Checkers), news (News Media), other people (Public), and computers (AI). All were worded to be politically neutral. Note that the four indicators include two common credible news sources (i.e., news outlets and fact checking sites) as well as two commonly proposed techniques for handling scale (i.e., crowdsourcing and computational analyses). For any non-true headlines encountered in the treatment conditions corresponding to each of the indicators, participants were shown the descriptive text for the respective indicator.

For each of the 12 presented headlines, participants were asked to indicate whether they would share the corresponding article with their friends on social media. After collecting the sharing intent for the 12 headlines, the responses were echoed back asking for reasons for the previously indicated sharing intent for each presented headline. We listed a set of reasons along

\author{
Coiled mattresses cause cancer by \\ amplifying radio waves \\ Multiple fact-checking journalists dispute the credibility of this news \\ Would you share this headline with your friends on social media? \\ $\mathrm{O}$ Yes \\ No
}

Figure 1: Screenshot showing an Obscure False headline presented in the study with the Fact Checkers indicator.

with the option to specify other factors. The listed choices were taken from Ma et al. [32] with minor modifications to fit our study. For the treatment conditions, we provided an additional choice to indicate the influence of the encountered credibility indicators.

Finally, we asked for demographics and other relevant information, such as social media use and news consumption. In the treatment conditions, we additionally asked whether and how participants used the encountered credibility indicators.

\section{Headline Selection}

The semantics of a headline play a notable role in the likelihood of the corresponding article being shared with others. To minimize disproportionate impact of any single type of headline and ensure broad applicability, we sought to use headlines covering a diverse variety of topics across three categories of varying credibility: true news, falsehoods, and satire. While falsehoods and satire are both factually untrue, the intentions of satire creators are benign because it does not deliberately aim to sow and spread misinformation with malicious intent. It is therefore important to study whether people distinguish between the two forms of non-true content.

Within each of the three types, we included well-known as well as obscure stories to vary the likelihood of someone having previously encountered the headline. For instance, Well-known False headlines were chosen from stories widely reported as fake by reputed media outlets such as The New York Times, CNN, The Guardian, etc. On the other hand, Obscure False headlines were chosen from those that did not receive such widespread fact checking attention. Similarly, Well-known Satire headlines were taken from popular satirical sources such as The Onion and The Borowitz Report (a satire column in the New Yorker) while Obscure Satire headlines were collected from lesser known sources. Where applicable, we used independent fact checking services, such as https://snopes.com, https://politifact.com, etc., to check the credibility of the stories connected to the headlines.

We collected headlines at two different times during the year to avoid oversampling headlines relevant only to current trends. Our initial collection included more than 200 headlines on various topics across the three categories described above. To ensure adequate statistical power, each headline needed to be shown to a reasonable number of participants. Therefore, we trimmed the initial set by removing headlines older than a year and not relevant to the U.S. context. From the trimmed set, 
we chose 72 headlines for inclusion in the study by majority voting among three of the authors such that there were 24 headlines in each of the three categories (True, False, and Satire), further divided into 12 Well-known and 12 Obscure per category. The 72 headlines cover a variety of topics such as politics, science, entertainment, everyday events, etc. (See Supplementary Material for the full set). Some examples of the headlines are: Tens of thousands of fraudulent Clinton votes found in Ohio warehouse (Well-known False), Coiled mattresses cause cancer by amplifying radio waves (Obscure False), Researchers Identify Gene for Awfulness (Well-known Satire), Geologists Say Continents May Have Drifted Apart After Emotional Falling-Out (Obscure Satire), 200,000 people apply to live on Mars (Well-known True), Google no longer lets you change domains to search different countries (Obscure True).

\section{Study Deployment}

We iteratively refined the study via three in-person pilots at our institutions. Feedback from pilot participants helped us tailor the list of reasons for sharing intent, estimate minimum and average times required to complete the study, and uncover and fix bugs in study flow. Additionally, the pilots helped confirm the validity and credibility of the study design.

We advertised the study as a Human Intelligence Task (HIT) on the Amazon Mechanical Turk (AMT) crowdwork platform for a period of 16 weeks from mid-May to early September 2018. The HIT provided a link to the online study. We limited participation to those 18 years of age or older based in the United States with more than 50 approved HITs and a HIT approval rating of at least $95 \%$. Those who visited the link were randomly assigned to one of the five study conditions (control or one of the four treatment conditions corresponding to one of the four credibility indicators).

We included several measures within the study to ensure collection of high quality responses. At the beginning, we included a screening question asking participants if they intended to provide high quality answers. Only those who answered in the affirmative were allowed to proceed; others were asked to return the HIT on AMT. Within the study, we included an attention check question that asked participants to select the answer to a simple mathematical addition. Further, we included a manipulation check that asked people for reasons for sharing a headline that was not part of the study and not shown to the participants for indicating their sharing intent. In addition, we set a browser cookie to reduce the chances of multiple submissions by the same person, and we ensured that no AMT worker completed the study more than once.

At the end of the study, participants received a unique randomly generated code to be entered on AMT to receive compensation for completing the study. All those who entered the correct code and passed the attention check received a compensation of $\$ 1$ for completing the study even if they failed the manipulation check. With a median study completion time of 10 minutes, the compensation translates to $\$ 6 /$ hour which is typical for AMT based studies. All study procedures were reviewed and approved by the Institutional Review Boards
(IRBs) of the institutions of the authors. (See Supplementary Material for the complete study instrument and procedures.)

\section{Sample}

A total of 2,803 individuals completed the study by submitting a completion code on AMT. Of these, we excluded those who failed the attention check and/or the manipulation check described earlier and those with non-U.S. IP addresses. The median study completion time was 10 minutes. We therefore excluded those who completed the study in less than five minutes, which was substantially below the median completion time as well as the completion times observed during pilots. Note that these filtering criteria were set prior to data collection and applied prior to data analysis.

After filtering, we ended up with valid data from 1,512 participants with ages ranging from $18-78$ (mean $=38$; median $=35$ ) and genders distributed fairly evenly among females and males, with $49 \%(\mathrm{~N}=735)$ females and $51 \%(\mathrm{~N}=768)$ males. Five participants marked their genders as 'Other,' and four chose not to reveal their respective genders. Participants included individuals who were employed $(74 \% ; \mathrm{N}=1,114)$, students ( $3 \% ; \mathrm{N}=38)$, retired ( $3 \% ; \mathrm{N}=39)$, unemployed (6\%; $\mathrm{N}=86)$, and others (16\%; $\mathrm{N}=235)$.

Overall, the 1,512 participants were shown 18,144 headlines across all conditions resulting in 18,135 responses regarding sharing intent and 9 instances where the participant chose not to answer. The 1,512 participants were distributed nearly uniformly across the five study groups: 299 in the control condition and 309, 305, 291, and 308 in the Fact Checkers, News Media, Public, and AI treatment conditions, respectively.

\section{Analysis Approach}

Our outcome variable was the intent to share the article corresponding to a headline with friends on social media. Since the outcome variable is a binary choice between the intent to share or not share, we employed Binomial Logistic Regression (BLR). The BLR incorporated participant ID and headline ID as random effects to account for repeated measures for varying items $[5,68]$. Our independent variables included the treatment, headline category, political affiliation, social media use, and common demographic factors, such as age and gender.

We used the stepwise approach described by Winter [68] and others [63] to incorporate the various independent variables of interest along with corresponding interaction effects. We followed standard practices for model selection and significance testing for mixed models by using the Likelihood Ratio Test (LRT) that tests the difference between two nested models using the Chi-square test. First, we compared the initial constant model (with no predictors) with the model that includes predictors. We retained a predictor as long as it exhibited statistical significance compared to the initial model.

Additionally, we analyzed the responses to open-ended questions. Open-ended responses regarding intention to share or not share each headline were provided by those who chose "Other" as one of the reasons, which included a text field for explanation. The other two questions that solicited open-ended responses were: (i) Please tell us whether and how you utilized 
Table 1: Distribution of sharing intent for True and Non-true headlines across study conditions.

\begin{tabular}{lccc}
\hline $\begin{array}{c}\text { Study } \\
\text { condition }\end{array}$ & True headlines & Non-true headlines & $\begin{array}{c}\text { \% Difference from Control } \\
\text { for Non-true headlines }\end{array}$ \\
\hline Control & $398(33 \%$ of 1194$)$ & $586(25 \%$ of 2392$)$ & 0 \\
Fact Checkers & $401(32 \%$ of 1236$)$ & $345(14 \%$ of 2469$)$ & -43 \\
News Media & $414(34 \%$ of 1219$)$ & $450(18 \%$ of 2440$)$ & -25 \\
Public & $380(33 \%$ of 1164$)$ & $444(19 \%$ of 2327$)$ & -22 \\
AI & $387(31 \%$ of 1231$)$ & $472(19 \%$ of 2463$)$ & -22 \\
\hline
\end{tabular}

the red text below the headline regarding the credibility of the news (whenever such information was available); and (ii) Is there anything else you would like to tell us? The third author analyzed a sample of open-ended responses and developed a codebook of salient themes in the answers. The codebook was refined in consultation with the last author. Three coders used the codebook to code a small set of open-ended responses. The third author was one of the coders while the other two were unconnected to the research. Initial coding revealed a large number of discrepancies among the three coders which were resolved by an in-depth in-person discussion to reach consensus and refine the codebook. The refined codebook was applied to code the rest of the open-ended responses, reaching much higher levels of agreements (ranging from 78\% to 85\% of cases marked the same by all three coders, depending on the question). All discrepancies were marked and resolved via discussion until the three coders reached consensus.

\section{FINDINGS}

Table 1 shows the sharing intent indicated by the participants for the various headlines across all study conditions, with False and Satire headlines grouped as Non-true headlines. The table indicates that the sharing intent for True headlines was similar across all conditions while that for Non-true headlines differed from the control condition. We report the statistical significance and size of these effects based on analyses that follow the approach outlined in the Method section.

As mentioned in Method Section, we did not include credibility indicators for any True headlines even in the treatment conditions. However, the presence of credibility indicators for the other headlines in the treatment conditions could conceivably have impacted the sharing intent for True headlines in those conditions. Therefore, we first examined the sharing intent for only the True headlines in all treatment conditions combined, compared with the True headlines in the control condition as the baseline. Unlike the findings of Pennycook et al. [43], we found no statistically significant differences when comparing the control condition against all treatment conditions combined $(\beta=0.02421, \mathrm{SE}=0.1216, \mathrm{p}=0.842)$ as well as separated individually: Control vs. Fact Checkers $(\beta=-0.01973, \mathrm{SE}=0.15304, \mathrm{p}=0.897)$, Control vs. News Media $(\beta=0.14246, \mathrm{SE}=0.15277, \mathrm{p}=0.351)$, Control vs. Public $(\beta=0.04717, \mathrm{SE}=0.15483, \mathrm{p}=0.761)$, Control vs. $\mathrm{AI}(\beta=-0.07233, \mathrm{SE}=0.15298, \mathrm{p}=0.636)$. This lack of a difference is also apparent in Table 1 with the percentages of True headlines participants intended to share being roughly the same across all conditions.

Given the lack of a statistical difference among study conditions for the intention to share True headlines, we limited

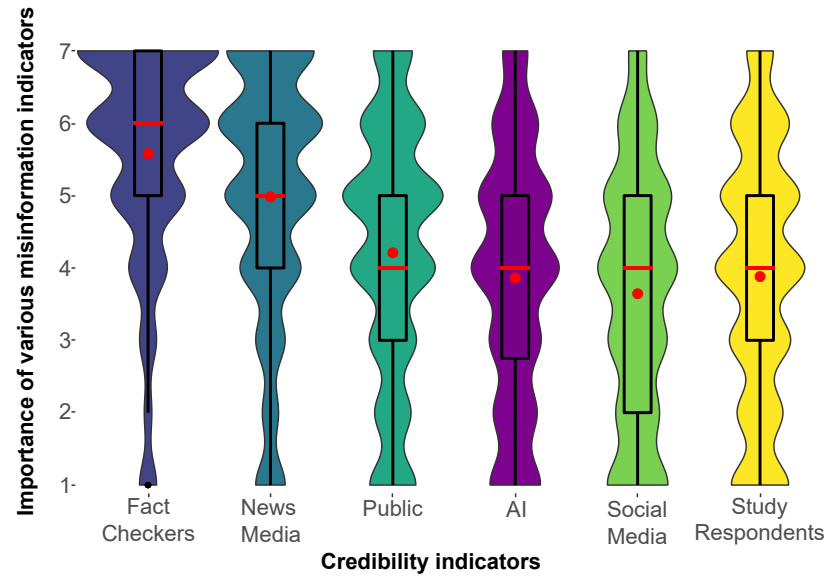

Figure 2: Violin plots showing self-reported importance of the various credibility indicators on a scale of 1 (Not at all important) to 7 (Extremely important). Each violin plot contains the corresponding box and whiskers plot showing the median with a red bar and the mean with a red dot.

subsequent BLR steps to the two Non-true types of headlines in our study, i.e., False and Satire. For these two types, those in the control condition saw no credibility indicators while those in the treatment conditions saw one of the four credibility indicators described in the Method section, accounting for 12,096 decisions for sharing intent across all conditions.

Since we have a large number of independent variables, we checked for correlations among these variables and discarded the less important ones that correlated with the more important predictors. We then followed a stepwise approach, progressively adding predictors at each step while removing those found to be statistically non-significant in the previous step. Where relevant, we tested for interaction effects among the predictors. As mentioned earlier, each step included the participant ID and headline ID as random effects to account for repeated measures for varying items $[5,68]$. The first step examined the effect of the study conditions followed by the headline type, political affiliation, and demographics and social media use, respectively. Across all study conditions, we found no differences in sharing intent between False and Satire headlines. Therefore, in subsequent analyses, we considered them together as Non-true headlines. Similar results were obtained when considering each separately. The final model without interaction effects resulting at the end of the last step of the BLR is shown in Table 2. Complete regression results including those with interaction effects are included in Supplementary Material. The following subsections discuss the impact of each of the factors included in the model in turn, followed by a discussion of participant responses for reasons behind their sharing intent.

\section{Effect of Credibility Indicators}

In the case of Non-true headlines, we found a statistically significant difference in sharing intent expressed in all of the treatment conditions combined when compared with that in the control condition. Participants in the control condition 
Table 2: Last step of the stepwise Binomial Logistic Regression (BLR) showing the final model for the intent to share news on social media.

\begin{tabular}{|c|c|c|c|c|}
\hline Variable & Odds ratio & $2.5 \%$ & $97.5 \%$ & $\mathbf{p}$ \\
\hline $\begin{array}{l}\text { Baseline: } \text { Condition = Control } \\
\text { Condition = Treatment }\end{array}$ & 0.397 & 0.297 & 0.531 & $1.95 e-07^{* * *}$ \\
\hline \multicolumn{5}{|c|}{$\begin{array}{l}\text { Baselines: } \\
\text { Condition }=\text { Control, Political Affiliation }=\text { Democrat }, \text { Gender }=\text { Female, } \\
\text { Age }=18-35, \text { Social Media Usage }=\text { Low }\end{array}$} \\
\hline Condition $=$ Fact Checkers & 0.204 & 0.124 & 0.335 & $3.59 e-10^{* * *}$ \\
\hline Condition $=$ News Media & 0.446 & 0.306 & 0.649 & $2.51 e-05^{* * *}$ \\
\hline Condition $=$ Public & 0.502 & 0.334 & 0.752 & $0.000856^{* * *}$ \\
\hline Condition $=\mathrm{AI}$ & 0.485 & 0.330 & 0.714 & $0.000244^{* * *}$ \\
\hline Political Affiliation = Independent & 1.312 & 1.022 & 1.686 & $0.033388^{*}$ \\
\hline Political Affiliation = Republican & 2.658 & 1.957 & 3.610 & $3.92 e-10^{* * *}$ \\
\hline Gender $=$ Male & 1.481 & 1.180 & 1.860 & $0.000725^{* * *}$ \\
\hline Age $=(35+)$ & 0.513 & 0.409 & 0.642 & $6.31 e-09^{* * *}$ \\
\hline Social Media Usage $=$ High & 2.172 & 1.658 & 2.847 & $1.84 e-08^{* * *}$ \\
\hline
\end{tabular}

intended to share a quarter of the 2,392 Non-true headlines they encountered. Across all treatment conditions combined, participants intended to share a much smaller proportion (18\%) of the 9,699 Non-true headlines shown to them (see Table 1). The odds ratio of the intention to share Non-true headlines in the treatment conditions compared to control was 0.397 , with the 95\% confidence interval (CI) between 0.297 and 0.531 ( $\mathrm{p}<0.001)$. In other words, the odds of the intention to share Non-true headlines were 2.52 times less in the treatment conditions compared to control, with the $95 \%$ CI between 1.88 and $3.37(\mathrm{p}<0.001)$. The statistically significant decrease in the treatment conditions demonstrates that the presence of a credibility indicator contributed to reduced sharing intent for Non-true articles.

Table 1 shows that the distribution of responses for the Nontrue headlines differed across the four treatment conditions. The Fact Checkers indicator was the most successful in persuading people to avoid sharing Non-true headlines, with a percentage decrease of $43 \%$ in sharing percentage compared to the control condition, while the AI indicator was the least effective with a corresponding percentage decrease of $22 \%$. The News Media and Public indicators led to slightly greater decreases than those with the AI indicator. When compared with the control condition as the baseline, the $95 \%$ CIs of the News Media, Public, and AI indicators do overlap with each other. However, with the control condition as the baseline, the 95\% CI for the Fact Checkers indicator did not overlap with those of any other indicator. Tukey's tests confirmed that the Fact Checkers treatment group was statistically significantly different from the control group as well as the other treatment groups (see Table 3$)^{2}$. The odds of sharing intent were reduced at least 2.188 times by the Fact Checkers indicator compared to the other indicators $(\mathrm{p}<0.05)$, as reflected by the odds ratio of 0.457 between Fact Checkers and News Media, which was the next most effective indicator.

Toward the end of the study, we asked all participants to rate the importance of each of the four credibility indicators on a scale of 1 to 7, with 1 indicating 'Not at all important' and

\footnotetext{
${ }^{2}$ Similar results were obtained by testing the equality of coefficients of the respective treatment groups in the regression model.
}

Table 3: Results of Tukey's test comparing various study conditions with each other.

\begin{tabular}{lcccc}
\hline Groups & Odds ratio & $\mathbf{2 . 5 \%}$ & $\mathbf{9 7 . 5 \%}$ & $\mathbf{p}$ \\
\hline Fact Checkers / Control & 0.204 & 0.124 & 0.335 & $<0.0001^{* * *}$ \\
News Media / Control & 0.446 & 0.306 & 0.649 & $0.0002^{* * *}$ \\
Public / Control & 0.502 & 0.334 & 0.752 & $0.0076^{* *}$ \\
AI / Control & 0.485 & 0.330 & 0.714 & $0.0023^{* *}$ \\
Fact Checkers / News Media & 0.457 & 0.268 & 0.777 & $0.0313^{*}$ \\
Fact Checkers / Public & 0.406 & 0.235 & 0.701 & $0.0107^{*}$ \\
Fact Checkers / AI & 0.419 & 0.246 & 0.716 & $0.0126^{*}$ \\
News Media / Public & 0.889 & 0.579 & 1.370 & 0.9833 \\
News Media / AI & 0.919 & 0.604 & 1.400 & 0.9948 \\
Public / AI & 1.015 & 0.673 & 1.659 & 0.9999 \\
\hline \multicolumn{4}{c}{ Reported p values are corrected to account for multiple pairwise comparisons. }
\end{tabular}

7 indicating 'Extremely important.' We further included two indicators not shown during the treatment: "A majority of your social media friends disputes the credibility of this news." (Social Media) and "A majority of study respondents disputes the credibility of this news." (Study Respondents). These responses enabled us to compare the observed sharing intent with self-reported preferences (see Figure 2). We further analyzed open-ended responses from participants in the treatment conditions regarding their use of the credibility indicators they encountered. We found that more than half $(52 \%)$ of the participants in the treatment conditions indicated that they consulted the credibility indicators while $36 \%$ of the participants explicitly stated that they did not use the indicators.

\section{Effect of Political Affiliation}

Upon initial inspection, we found that political ideology, political affiliation, and political registration were highly correlated. Further, we found no notable differences between those with strong or weak political alignment, either Democrat or Republican. Therefore, we grouped those who expressed either weak or strong political alignment for either party, thus ending up with three kinds of political affiliation: Democrat (39\%), Republican (20\%), and Independent (41\%). Upon including political affiliation in the BLR, we observed that Republicans and Independents intended to share Non-true headlines to a much greater extent compared to Democrats (see Table 2), with odds ratios of 2.658 and $1.312(p<0.001$ and $p=0.033)$, respectively. When considering interaction effects, we found that the presence of indicators contributed to reducing the sharing intent of Democrats the most, followed by Independents and Republicans (see Figure 3a). However, for Republicans, the differences in sharing intent for Non-true headlines were not statistically significant in comparison to the control condition. (Regression results of the model with interaction effects are included in Supplementary Material.)

The Fact Checkers indicator remained the most effective regardless of political affiliation. The effects of all indicators, however, differed according to political affiliation. Democrats exhibited the highest reduction in sharing intent for Non-true headlines, and Republicans were affected the least; Independents fell somewhere between the two (see Figure 3a). As Figure 3a shows, in the case of Republicans, the Public and News Media indicators exhibited much smaller effects and the 


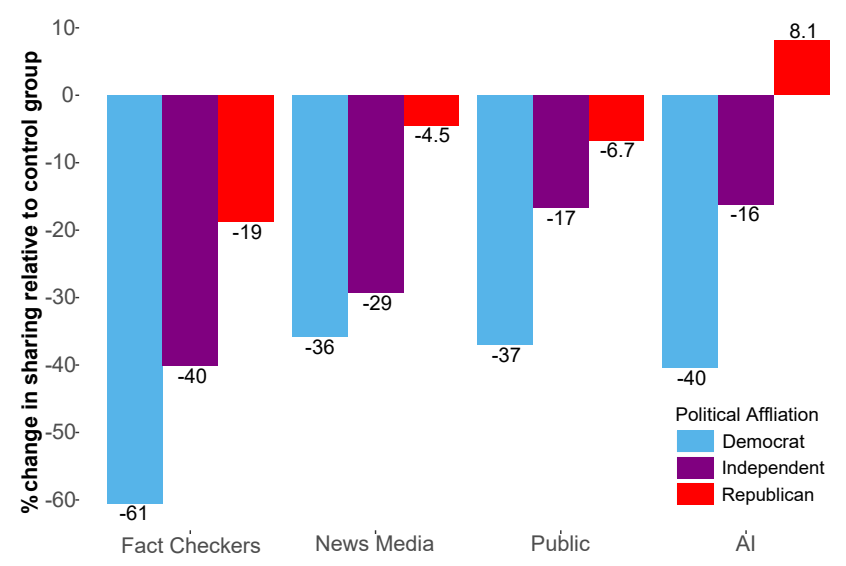

(a) Political Affiliation

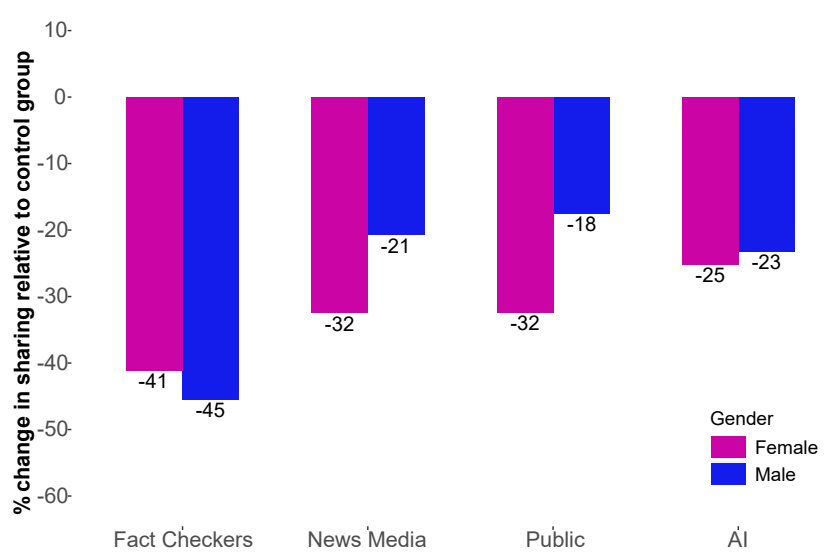

(b) Gender

Figure 3: Changes in percentages of sharing intent across treatment groups separated by political affiliation and gender.

AI indicator led to an increase in Non-true headline sharing intent compared to the control condition.

\section{Effect of Demographics and Social Media Usage}

Finally, we examined the influence of common demographics (i.e., gender and age) and self-reported social media usage.

Overall, we found that females responded more to the credibility indicators. When compared with the control condition, a Tukey test showed that the odds of sharing intent for Non-true headlines by males in all treatment conditions combined were 1.59 times more than those for females $(p=0.0062)$. When examining the treatment conditions separately, we found that the differences were the largest for the Public and News Media indicators whereas the Fact Checkers indicator was slightly less effective on females compared to males (see Figure $3 b$ ).

Our initial analyses revealed no notable differences among those in age ranges between $18-35$ (52\% of the sample) and among those above 35 (48\% of the sample). Therefore, we examined the impact of age by splitting the sample at age 35 . Those older than 35 intended to share Non-true headlines to a lesser extent (see Table 2). However, a Tukey test showed that both age groups were influenced by the Fact Checkers indicator, with odds ratios for sharing intent for the Fact Checkers condition compared to the control condition being 0.217 and 0.140 for the $18-35$ and $35+$ age groups, respectively $(p<$ $0.001)$.

Those who reported higher use of social media intended to share more of the Non-true headlines (see Table 2). However, we noted that the Fact Checkers indicator exhibited a large impact regardless of whether the use of social media was reported to be high or low. When analyzing the impact of the Fact Checkers indicator on sharing intent in comparison to the control condition, a Tukey test showed that high and low use of social media corresponded to odds ratios of 0.180 ( $<<$ $0.001)$ and $0.184(\mathrm{p}=0.0039)$, respectively.

\section{Reasons of Sharing}

To understand the rationales behind sharing intent, we examined the reasons provided by the participants for their choices. Figure 4 shows plots of the percentages for the various reasons for the intent to share or not share in the control and treatment conditions, respectively. As the figure shows, across all conditions, interestingness and potential for generating conversation were the dominant reasons for intending to share and uninterestingness and irrelevance were the dominant reasons intending not to share, regardless of the veracity of the news.

In the control as well as treatment conditions, a notable proportion of the participants intended not to share Non-true headlines because of their false nature. That said, falseness as a reason for not sharing was provided $7 \%$ more in the treatment conditions than in the control condition $(p=0.00565)$, thus suggesting that the credibility indicators provided a boost to credibility judgments. The usefulness of the indicators for judging credibility was confirmed by the participants as well; $30 \%$ of the times when the participants decided not to share a Non-true headline, they marked the reason as: 'I was influenced by the red text below the headline regarding the credibility of the news."

\section{DISCUSSION AND IMPLICATIONS}

Although our exclusions were pre-determined and systematic, the relatively high proportion of exclusions warrants discussion. Out of 2,803 people who tried to complete the study: (i) 128 did not finish, thus could not be included; (ii) 139 answered in less than 5 minutes, which would not allow sufficient time for responding meaningfully; (iii) 308 were not from the United States (as determined by IP addresses); (iv) 3 participants did not agree to provide best answers, thus selfprofessing to potential inattention; (v) 816 failed the subtle manipulation check; and (vi) 36 failed the attention check despite passing the manipulation check. We flagged those who failed attention and/or manipulation checks as inattentive and dropped them from analysis [2]. It should be noted that attention and manipulation checks were administered toward the end of the study after collecting the main data of interest. 

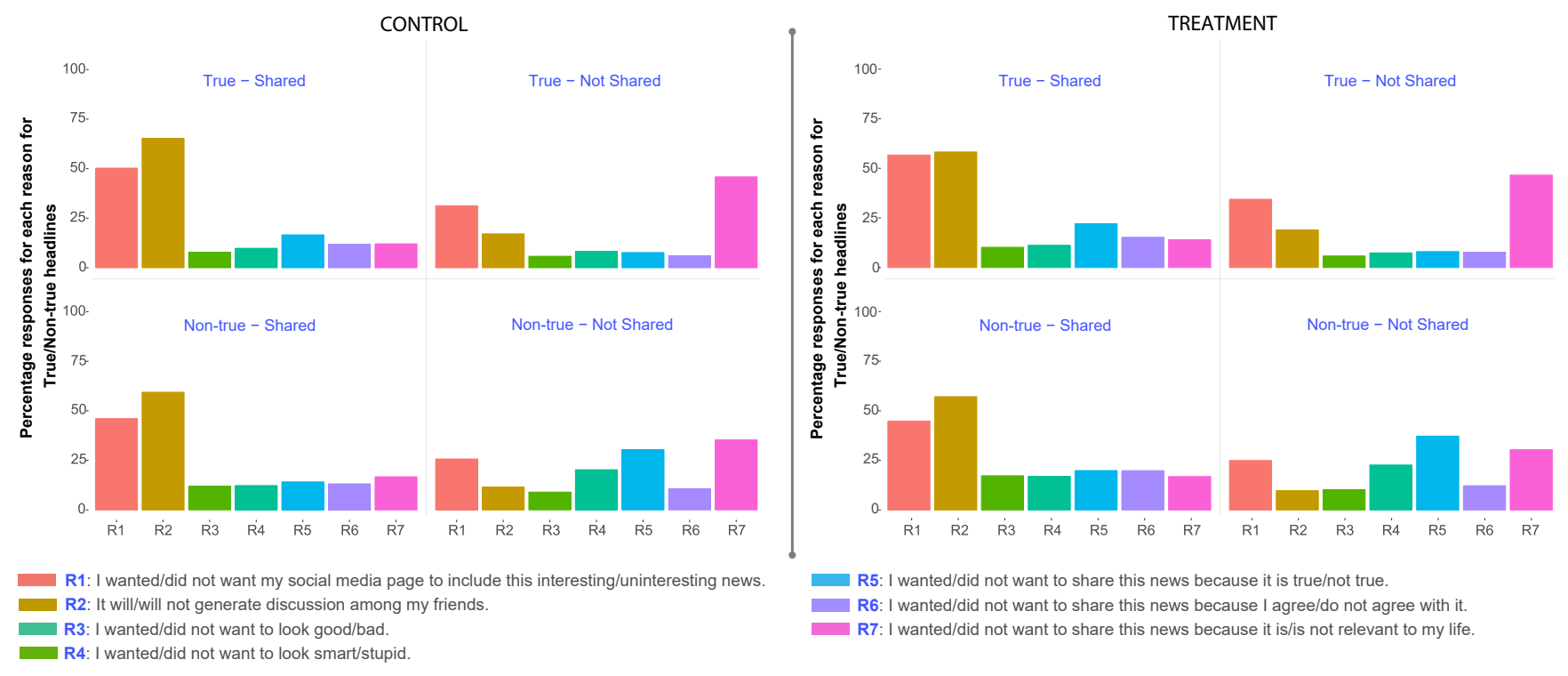

Figure 4: Reasons specified for intent to share or not share in the control and treatment conditions, respectively.

As such, any influence of the presence of the checks could not have affected responses to any questions except demographics.

Since we were working with a priori hypotheses operationalized via our independent and dependent variables, a correction was not required for the main analyses. We applied appropriate corrections to adjust the $\mathrm{p}$-values when making pairwise comparisons via Tukey's test. Notably, we found that all major factors covered by our research questions exhibited statistically significant effects on the intent to share non-true news. Although our study setting differs from a real-world social media platform, our analyses involved comparing across conditions in which only the credibility indicator varied. Therefore, the impact of differences with a real-world scenario can be expected to be distributed evenly across the study conditions, thus being nullified in comparative analyses. In other words, the differences we observed are independent of the implementation details of social media platforms. Moreover, the observed behavior (i.e., sharing intent) of the participants matched their self-reported preferences (i.e., ratings for the types of credibility indicators), lending further credibility to our findings. While we believe that the effects can generalize to real-world social media platforms, the baselines and effect sizes are likely to differ owing to operational differences.

While our credibility indicators did not always succeed in suppressing the intent to share Non-true headlines, they did lead to a sizable decrease in the proportion of such headlines that participants intended to share. Given the scale and amplification effects of social media, even a small reduction in sharing at the beginning of the chain is likely to result in substantive changes in the overall amount of sharing across the platform. For the most part, the mere presence of a credibility indicator of any kind served to decrease the propensity to share Non-true headlines. Moreover, our results show that the influence of our credibility indicators on reducing sharing intent applies across the various categories of non-true news.
Based on the individual characteristics of the participants, some indicators were more effective than others. The Fact Checkers indicator was a notable exception as it was found to be the most effective regardless of the influence of other factors. These findings suggest that independent fact checkers have an important role to play in solutions to help people recognize false stories, especially since they can be a nonpartisan influence, thus appealing to individuals across the political spectrum. How to allocate and scale high quality fact checking resources within the news sharing user experience is likely to be an important area for future research.

Republicans seemed to be the least influenced by the News Media indicator which is in line with polls and studies of the U.S. population that show Republicans being distrustful of mainstream media outlets $[23,29,45,61]$. Similarly, the Public indicator impacted Republicans to a much smaller extent compared to Democrats and Independents. It would be interesting to explore whether the impact of the Public indicator can be improved by using affinity groups so that Republicans are informed regarding the judgments of other Republicans rather than Americans as a whole. However, such an approach should be used with caution as it could create and reinforce echo chambers that are disconnected from the veracity of the content.

In terms of demographics, the higher sharing intent for Nontrue headlines by males could likely be attributed to males exhibiting a higher tendency to share political news compared to females [13]. Similarly, the higher sharing intent for nontrue headlines by younger participants may be due to their comparatively higher levels of social media engagement and content sharing compared to those who are older.

The dominant reasons for intending to share a headline were connected with socializing, thus echoing previous findings on motivations for social media content sharing [30]. In open- 
ended responses, the top reported reason for intending to share fake stories was that they were funny. Unless a user chooses to include an explicit comment indicating such reasons for knowingly sharing non-true content, the simple action of sharing cannot distinguish the user from those who share because they believe the news and support the expressed views. It could therefore be useful to design efficient UI mechanisms to help users annotate sharing acts with their intent, without requiring them to enter long text comments or explanations.

The goal of the study was to evaluate the impact of the presence and the content of an indicator, independent of the manner in which the credibility information is delivered and embedded in the underlying platform. Therefore, we displayed headlines that lacked the personalization of social media platforms. Study participants encountered a randomly chosen subset from our curated set of headlines. It is therefore unsurprising that in about $30 \%$ of cases across all study conditions, participants intended not to share because they found the headline uninteresting or irrelevant, regardless of veracity (see Figure 4). In such cases, participants did not even need the credibility indicator. Such situations are significantly less likely in a realworld social media setting where content is typically heavily personalized to the user. Personalization should arguably raise the effectiveness of our credibility indicators in a real-world setting where the encountered headlines are likely to be personally relevant and interesting.

The personalization capabilities of social media platforms could conceivably customize the indicators themselves by taking into account the above described differences based on individual characteristics. For instance, the content and delivery mechanism of the indicator could be varied based on gender. In addition, users could be allowed to specify preferences to select and customize the types of credibility indicators they desire. To that end, there is scope for exploring the design space for the content and delivery of credibility indicators. For instance, indicators could be enhanced by including details on the fact checking processes and the rationale for marking the news as false.

Our resources limited us to studying only four indicators. Future work could explore additional indicators, such as social categories of an order lower than the general public. In fact, as mentioned in the Findings section, when asking participants about the importance of various indicators, we included a couple of additional indicators.

It should be emphasized that our goal was limited to exploring the efficacy of credibility indicators as a UI mechanism for combating online misinformation by helping reduce its spread on social media. Determining how indicators are assigned to content involves important social, technical, and political considerations that are beyond the scope of this paper.

\section{LIMITATIONS}

Several limitations may affect the generalizability of our findings. The headlines we selected were published and available online. As a result, it is conceivable that participants may have encountered one or more of the headlines prior to study participation, thereby affecting sharing intent. We carefully worded the indicators in a politically neutral way. However, we cannot rule out the influence of inherent systemic political biases regarding technology or the media.

While insight from studies involving AMT workers is considered reasonably close to that from a sample more representative of the general population, AMT workers are known to have comparatively higher levels of information literacy and technical expertise. These characteristics could have impacted credibility judgment and sharing intent. Moreover, our sample and study context was limited to the United States. Therefore, replication of the study in other cultural contexts is necessary to confirm the generalizability and robustness of these findings. Further, future studies should investigate headlines beyond the set included in our study.

Participants indicated their sharing intent based only on the headline. It is possible that the sharing intent was impacted by the lack of additional information, such as the news source, the content of the article connected to the headline, etc. However, as mentioned in the Related Work section, prior studies have found that it is common for people to share news based only on the headline and similarly common not to take into account the article source.

Unlike the real world, our study setting presented a hypothetical scenario that held no social consequences for the participants. The interface and the user experience differed from a real-world setting as well. Deployment on a social media platform is necessary to verify the extent to which our findings translate to real-world operation.

\section{CONCLUSION}

Spread of misinformation via online communication platforms has become a global phenomenon with grave sociopolitical implications. Countering misinformation necessarily requires a multi-faceted approach that integrates a number of solutions, each making a contribution to the online information dissemination ecosystem. We explored one such aspect by targeting the human element in the system. Specifically, we examined whether we could help dampen and reduce the spread of misinformation by annotating it with credibility indicators. Additionally, the indicators can contribute to increasing information literacy by helping users get better at recognizing legitimate content. We found that credibility indicators can indeed serve to combat fake news. However, their effectiveness varies based on the type of indicator and personal characteristics of the user. Our findings are the first step in a design exploration of content and delivery of credibility markers and raise important sociopolitical questions regarding how content credibility could be evaluated, enacted, and enforced.

\section{ACKNOWLEDGMENTS}

We thank those who participated in our study and the preceding pilot. We acknowledge the help of Jacob Abbott and Emma Lashley in coding the open-ended responses. Thanks are due to Judy Kay, Mihir Mahajan, and anonymous reviewers for their feedback that helped improve the paper. 


\section{REFERENCES}

[1] Hunt Allcott and Matthew Gentzkow. 2017. Social Media and Fake News in the 2016 Election. Journal of Economic Perspectives 31, 2 (May 2017), 211-236. DOI : http://dx.doi.org/10.1257/jep.31.2.211

[2] R. Michael Alvarez, Lonna Rae Atkeson, Ines Levin, and Yimeng Li. 2019. Paying Attention to Inattentive Survey Respondents. Political Analysis 27, 2 (2019), 145-162. DOI : http://dx.doi.org/10.1017/pan.2018.57

[3] Chris Baraniuk. 2018. How Twitter bots help fuel political feuds. https://www.scientificamerican.com/ article/how-twitter-bots-help-fuel-political-feuds/, Scientific American (March 2018). (Retrieved January 7 , 2020).

[4] Michael Barthel, Amy Mitchell, and Jesse Holcomb. 2016. Many Americans believe fake news is sowing confusion.

https://www.journalism.org/wp-content/uploads/sites/8/ 2016/12/PJ_2016.12.15_fake-news_FINAL.pdf, Pew Research Center (December 2016). (Retrieved January 7, 2020).

[5] Markus Brauer and John J. Curtin. 2018. Linear mixed-effects models and the analysis of nonindependent data: A unified framework to analyze categorical and continuous independent variables that vary within-subjects and/or within-items. Psychological Methods 23, 3 (2018), 389-411. DOI:

http://dx.doi.org/10.1037/met0000159

[6] David A. Broniatowski, Amelia M. Jamison, SiHua Qi, Lulwah AlKulaib, Tao Chen, Adrian Benton, Sandra C. Quinn, and Mark Dredze. 2018. Weaponized Health Communication: Twitter Bots and Russian Trolls Amplify the Vaccine Debate. American Journal of Public Health 108, 10 (2018), 1378-1384. DOI: http://dx.doi.org/10.2105/AJPH.2018.304567

[7] Fernando Cardoso Durier da Silva, Rafael Vieira, and Ana Cristina Garcia. 2019. Can Machines Learn to Detect Fake News? A Survey Focused on Social Media. In Proceedings of the 52nd Hawaii International Conference on System Sciences (HICSS 2019). DOI: http://dx.doi.org/10.24251/HICSS.2019.332

[8] Paul Chadwick. 2017. How fares trust in journalism amid a sea of fake news? https:

//www.theguardian. com/commentisfree/2017/jul/09/howfares-trust-in-journalism-amid-a-sea- of-fake-news. (July 2017). (Retrieved January 7, 2020).

[9] Ziv Epstein, Gordon Pennycook, and David G. Rand. 2019. Will the crowd game the algorithm? Using layperson judgments to combat misinformation on social media by downranking distrusted sources. PsyArXiv Preprints (June 2019). DOI: http://dx.doi.org/10.31234/osf.io/z3s5k

[10] Henry John Farrell and Bruce Schneier. 2018. Common-Knowledge Attacks on Democracy. Berkman Klein Center Research Publication 2018-7 (October 2018). DOI : http://dx.doi.org/10.2139/ssrn. 3273111
[11] Miriam Fernandez and Harith Alani. 2018. Online Misinformation: Challenges and Future Directions. In Companion Proceedings of The Web Conference 2018 (WWW '18). International World Wide Web Conferences Steering Committee, Republic and Canton of Geneva, CHE, 595-602. DOI : http://dx.doi.org/10.1145/3184558.3188730

[12] Philip Fernbach and Sloman Steven. 2017. Why We Believe Obvious Untruths. https://www.nytimes.com/2017/03/03/opinion/sunday/whywe-believe-obvious-untruths.html. (March 2017). (Retrieved January 7, 2020).

[13] Fractl. 2016. Average Facebook User Sharing Habits Study. https://www.frac.tl/work/marketing-research/ facebook-user-sharing-habits-study/. (April 2016). (Retrieved January 7, 2020).

[14] Fraunhofer-Gesellschaft. 2019. Software that can automatically detect fake news. https://phys.org/news/ 2019-02-software-automatically-fake-news.html. (February 2019). (Retrieved January 7, 2020).

[15] Maksym Gabielkov, Arthi Ramachandran, Augustin Chaintreau, and Arnaud Legout. 2016. Social Clicks: What and Who Gets Read on Twitter? SIGMETRICS Performance Evaluation Review 44, 1 (June 2016), 179-192. DOI : http://dx.doi.org/10.1145/2964791.2901462

[16] John Hardwig. 1985. Epistemic Dependence. The Journal of Philosophy 82, 7 (1985), 335-349. DOI: http://dx.doi.org/10.2307/2026523

[17] Naeemul Hassan, Afroza Sultana, You Wu, Gensheng Zhang, Chengkai Li, Jun Yang, and Cong Yu. 2014. Data in, Fact out: Automated Monitoring of Facts by FactWatcher. Proceedings of the VLDB Endowment 7, 13 (Aug. 2014), 1557-1560. DOI : http://dx.doi.org/10.14778/2733004.2733029

[18] Alex Hern. 2018. New Facebook controls aim to regulate political ads and fight fake news. https://www.theguardian.com/technology/2018/apr/06/ facebook-launches-controls-regulate-ads-publishers. (April 2018). (Retrieved January 7, 2020).

[19] Benjamin D. Horne and Sibel Adali. 2017. This Just In: Fake News Packs a Lot in Title, Uses Simpler, Repetitive Content in Text Body, More Similar to Satire than Real News. In The Workshops of the Eleventh International AAAI Conference on Web and Social Media. Number WS-17-17: News and Public Opinion. AAAI Technical Report, 759-766.

[20] Seyedmehdi Hosseinimotlagh and Evangelos E. Papalexakis. 2018. Unsupervised Content-Based Identification of Fake News Articles with Tensor Decomposition Ensembles.

http://snap.stanford.edu/mis2/files/MIS2_paper_2.pdf, MIS2: Misinformation and Misbehavior Mining on the Web: Workshop held in conjunction with the 11th ACM International Conference on Web Search and Data Mining (February 2018). 
[21] Jacqueline Howard. 2019. Facebook debuts vaccine education pop-up windows. https://edition.cnn.com/2019/09/04/health/facebookvaccine-education-bn/index.html. (September 2019). (Retrieved January 7, 2020).

[22] Maurice Jakesch, Moran Koren, Anna Evtushenko, and Mor Naaman. 2018. The Role of Source, Headline and Expressive Responding in Political News Evaluation. (2018). DOI : http://dx.doi.org/10.2139/ssrn.3306403

[23] Jeffrey M. Jones. 2018. U.S. Media Trust Continues to Recover From 2016 Low. https://news.gallup.com/poll/ 243665/media-trust-continues-recover-2016-low.aspx. (October 2018). (Retrieved January 7, 2020).

[24] Dan M. Kahan, Ellen Peters, Maggie Wittlin, Paul Slovic, Lisa Larrimore Ouellette, Donald Braman, and Gregory Mandel. 2012. The polarizing impact of science literacy and numeracy on perceived climate change risks. Nature Climate Change 2, 10 (2012), 732-735. DOI : http://dx.doi.org/10.1038/nclimate1547

[25] Elihu Katz, Jay G. Blumler, and Michael Gurevitch. 1973. Uses and Gratifications Research. http://www.jstor.org/stable/2747854, The Public Opinion Quarterly 37, 4 (1973), 509-523.

[26] Jooyeon Kim, Behzad Tabibian, Alice Oh, Bernhard Schölkopf, and Manuel Gomez-Rodriguez. 2018. Leveraging the Crowd to Detect and Reduce the Spread of Fake News and Misinformation. In Proceedings of the Eleventh ACM International Conference on Web Search and Data Mining (WSDM '18). Association for Computing Machinery, New York, NY, USA, 324-332. DOI : http://dx.doi.org/10.1145/3159652.3159734

[27] Issie Lapowsky. 2018. This Browser Extension Is Like an AntiVirus for Fake Photos.

https://www.wired.com/story/surfsafe-browserextension-save-you-from-fake-photos/. (August 2018). (Retrieved January 7, 2020).

[28] David Lazer, Matthew Baum, Nir Grinberg, Lisa Friedland, Kenneth Joseph, Will Hobbs, and Carolina Mattsson. 2017. Combating fake news: An agenda for research and action. https://shorensteincenter.org/ combating-fake-news-agenda-for-research/. (May 2017). (Retrieved January 7, 2020).

[29] David M. J. Lazer, Matthew A. Baum, Yochai Benkler, Adam J. Berinsky, Kelly M. Greenhill, Filippo Menczer, Miriam J. Metzger, Brendan Nyhan, Gordon Pennycook, David Rothschild, Michael Schudson, Steven A. Sloman, Cass R. Sunstein, Emily A. Thorson, Duncan J. Watts, and Jonathan L. Zittrain. 2018. The science of fake news. Science 359, 6380 (2018), 1094-1096. DOI : http://dx.doi.org/10.1126/science.aao2998

[30] Chei Sian Lee and Long Ma. 2012. News sharing in social media: The effect of gratifications and prior experience. Computers in Human Behavior 28, 2 (2012), 331-339. DOI :

http://dx.doi.org/10.1016/j.chb.2011.10.002
[31] Yang Liu and Yi-Fang Brook Wu. 2018. Early detection of fake news on social media through propagation path classification with recurrent and convolutional networks. In Thirty-Second AAAI Conference on Artificial Intelligence (AAAI18).

[32] Long Ma, Chei Sian Lee, and Dion Hoe-Lian Goh. 2014. Understanding news sharing in social media : An explanation from the diffusion of innovations theory. Online Information Review 38, 5 (2020/01/12 2014), 598-615. DOI :

http://dx.doi.org/10.1108/0IR-10-2013-0239

[33] Jalal Mahmud and Huiji Gao. 2014. Why Do You Spread This Message? Understanding Users Sentiment in Social Media Campaigns. https://www.aaai.org/ocs/ index.php/ICWSM/ICWSM14/paper/view/8030/8090. In Eighth International AAAI Conference on Weblogs and Social Media.

[34] Adam Mosseri. 2017. Working to Stop Misinformation and False News. https://about.fb.com/news/2017/04/ working-to-stop-misinformation-and-false-news/. (April 2017). (Retrieved January 7, 2020).

[35] Dimitar Nikolov, Diego F. M. Oliveira, Alessandro Flammini, and Filippo Menczer. 2015. Measuring online social bubbles. PeerJ Computer Science 1 (Dec. 2015), e38. DOI : http://dx.doi.org/10.7717/peerj-cs.38

[36] Ben Nimmo. 2015. Anatomy of an Info-War: How Russia's Propaganda Machine Works, and How to Counter It. https://www.stopfake.org/en/anatomy-of-aninfo-war-how-russia-s-propaganda-machine-works-andhow-to-counter-it/. (May 2015). (Retrieved January 7, 2020).

[37] Brendan Nyhan, Ethan Porter, Jason Reifler, and Thomas J. Wood. 2019. Taking Fact-Checks Literally But Not Seriously? The Effects of Journalistic Fact-Checking on Factual Beliefs and Candidate Favorability. Political Behavior (21 January 2019), 1-22. DOI : http://dx.doi.org/10.1007/s11109-019-09528-x

[38] Brendan Nyhan and Jason Reifler. 2010. When Corrections Fail: The Persistence of Political Misperceptions. Political Behavior 32, 2 (2010), 303-330. DOI :

http://dx.doi.org/10.1007/s11109-010-9112-2

[39] Anne Oeldorf-Hirsch, Mike Schmierbach, Alyssa Appelman, and Michael P. Boyle. 2018. Credibility effects of disputed and confirmed information in social media news memes. In Proceedings of the 68th annual conference of the International Communication Association (ICA).

[40] Laura Hazard Owen. 2018. Crowdsourcing trusted news sources can work - but not the way Facebook says it'll do it. https://www.niemanlab.org/2018/02/crowdsourcingtrusted-news-sources-can-work-but-not-the-wayfacebook-says-itll-do-it/. (February 2018). 
[41] Laura Hazard Owen. 2019. Individually, people aren't great at judging news sources. En masse, they're almost the same as professional fact-checkers.

https://www.niemanlab.org/2019/02/individually-peoplearent-great-at-judging-news-sources-en-masse-theyrealmost-the-same-as-professional-fact-checkers/. (February 2019). (Retrieved January 7, 2020).

[42] Archita Pathak and Rohini Srihari. 2019. BREAKING! Presenting Fake News Corpus for Automated Fact Checking. https://www.aclweb.org/anthology/P19-2050. In Proceedings of the 57th Annual Meeting of the Association for Computational Linguistics: Student Research Workshop. Association for Computational Linguistics, Florence, Italy, 357-362.

[43] Gordon Pennycook, Adam Bear, Evan Collins, and David G. Rand. 2019. The Implied Truth Effect: Attaching Warnings to a Subset of Fake News Stories Increases Perceived Accuracy of Stories Without Warnings. Management Science (August 2019). DOI : http://dx.doi.org/10.2139/ssrn.3035384 (forthcoming).

[44] Gordon Pennycook and David Rand. 2019a. Why Do People Fall for Fake News? https://www.nytimes.com/ 2019/01/19/opinion/sunday/fake-news.html. (January 2019).

[45] Gordon Pennycook and David G. Rand. 2019b. Fighting misinformation on social media using crowdsourced judgments of news source quality. Proceedings of the National Academy of Sciences 116, 7 (2019), 2521-2526. DOI : http://dx.doi.org/10.1073/pnas.1806781116

[46] Gordon Pennycook and David G. Rand. 2019c. Lazy, not biased: Susceptibility to partisan fake news is better explained by lack of reasoning than by motivated reasoning. Cognition 188 (2019), 39-50. DOI : http://dx.doi.org/10.1016/j.cognition.2018.06.011

[47] Julio. C. S. Reis, André Correia, Fabrício Murai, Adriano Veloso, and Fabr'icio Benevenuto. 2019. Supervised Learning for Fake News Detection. IEEE Intelligent Systems 34, 2 (March 2019), 76-81. DOI : http://dx.doi.org/10.1109/MIS.2019.2899143

[48] Paul Resnick, Aviv Ovadya, and Garlin Gilchrist. 2019. Iffy Quotient: A Platform Health Metric for Misinformation. http://umsi.info/iffy-quotient-whitepaper. (July 2019). (Retrieved January 7, 2020).

[49] Timothy Revell. 2017. How to turn Facebook into a weaponised AI propaganda machine. https:

//www.newscientist.com/article/2142072-how-to-turnfacebook-into-a-weaponised-ai-propaganda-machine/. (July 2017). (Retrieved January 7, 2020).

[50] Manoel Horta Ribeiro, Pedro H. Calais, Virgílio A. F. Almeida, and Wagner Meira Jr. 2017. "Everything I Disagree With is \#FakeNews": Correlating Political Polarization and Spread of Misinformation. http://arxiv.org/abs/1706.05924, CoRR abs/1706.05924 (2017).
[51] Everett M. Rogers. 2003. Diffusion of Innovations (5th ed.). Free Press, New York, USA.

[52] Natali Ruchansky, Sungyong Seo, and Yan Liu. 2017. CSI: A Hybrid Deep Model for Fake News Detection. In Proceedings of the 2017 ACM on Conference on Information and Knowledge Management (CIKM '17). Association for Computing Machinery, New York, NY, USA, 797-806. DOI :

http://dx.doi.org/10.1145/3132847.3132877

[53] Scott Shackelford, Bruce Schneier, Michael Sulmeyer, Anne Boustead, Ben Buchanan, Amanda N. Craig Deckard, Trey Herr, and Jessica Malekos Smith. 2017. Making Democracy Harder to Hack.

https://repository.law.umich.edu/mjlr/vol50/iss $3 / 3$, University of Michigan Journal of Law Reform 50, 3 (January 2017), 629-668.

[54] Chengcheng Shao, Giovanni Luca Ciampaglia, Onur Varol, Alessandro Flammini, and Filippo Menczer. 2017. The spread of low-credibility contentby social bots. http://arxiv.org/abs/1707.07592, CoRR abs/1707.07592 (2017).

[55] Ian Sherr. 2018. Facebook and Twitter have new rules for political ads. Here's how they work.

https://www.cnet.com/news/facebook-and-twitter-havenew-rules-for-political-ads-heres-how-they-work/. (May 2018). (Retrieved January 7, 2020).

[56] Kai Shu, Amy Sliva, Suhang Wang, Jiliang Tang, and Huan Liu. 2017. Fake News Detection on Social Media: A Data Mining Perspective. SIGKDD Explorations Newsletter 19, 1 (Sept. 2017), 22-36. DOI : http://dx.doi.org/10.1145/3137597.3137600

[57] Craig Silverman. 2016. This Analysis Shows How Viral Fake Election News Stories Outperformed Real News On Facebook. https://www.buzzfeednews.com/article/ craigsilverman/viral-fake-election-newsoutperformed-real-news-on-facebook. (November 2016). (Retrieved January 7, 2020).

[58] Steven A. Sloman and Nathaniel Rabb. 2016. Your Understanding Is My Understanding: Evidence for a Community of Knowledge. Psychological Science 27, 11 (2016), 1451-1460. DOI:

http://dx.doi.org/10.1177/0956797616662271 PMID: 27670662.

[59] Kate Starbird, Jim Maddock, Mania Orand, Peg Achterman, and Robert M. Mason. 2014. Rumors, False Flags, and Digital Vigilantes: Misinformation on Twitter after the 2013 Boston Marathon Bombing. iConference 2014 Proceedings (2014), 654-662. DOI: http://dx.doi.org/10.9776/14308

[60] Massimo Stella, Emilio Ferrara, and Manlio De Domenico. 2018. Bots increase exposure to negative and inflammatory content in online social systems. Proceedings of the National Academy of Sciences 115, 49 (2018), 12435-12440. DOI :

http://dx.doi.org/10.1073/pnas.1803470115 
[61] Art Swift. 2016. Americans' Trust in Mass Media Sinks to New Low. https://news.gallup.com/poll/195542/ americanstrust-mass-media-sinks-new-low.aspx. (September 2016). (Retrieved January 7, 2020).

[62] Sebastian Tschiatschek, Adish Singla, Manuel Gomez Rodriguez, Arpit Merchant, and Andreas Krause. 2018. Fake News Detection in Social Networks via Crowd Signals. In Companion Proceedings of The Web Conference 2018 (WWW '18). International World Wide Web Conferences Steering Committee, Republic and Canton of Geneva, CHE, 517-524. DOI : http://dx.doi.org/10.1145/3184558.3188722

[63] University of Wisconsin Social Science Computing Cooperative. 2013. Mixed Models: Testing Significance of Effects. https :

//www.ssc.wisc.edu/sscc/pubs/MM/MM_TestEffects.html. (March 2013). (Retrieved January 7, 2020).

[64] Michela Del Vicario, Sabrina Gaito, Walter Quattrociocchi, Matteo Zignani, and Fabiana Zollo. 2017. Public discourse and news consumption on online social media: A quantitative, cross-platform analysis of the Italian Referendum. http://arxiv.org/abs/1702.06016, CoRR abs/1702.06016 (2017).

[65] Soroush Vosoughi, Deb Roy, and Sinan Aral. 2018. The spread of true and false news online. Science 359, 6380 (2018), 1146-1151. DOI :

http://dx.doi.org/10.1126/science.aap9559
[66] Helena Webb, Marina Jirotka, Bernd Carsten Stahl, William Housley, Adam Edwards, Matthew Williams, Rob Procter, Omer Rana, and Pete Burnap. 2016. Digital Wildfires: Hyper-Connectivity, Havoc and a Global Ethos to Govern Social Media. SIGCAS Computers and Society 45, 3 (Jan. 2016), 193-201. DOI :

http://dx.doi.org/10.1145/2874239.2874267

[67] Jen Weedon, William Nuland, and Alex Stamos. 2017. Information Operations and Facebook.

https://fbnewsroomus.files.wordpress.com/2017/04/ facebook-and-information-operations-v1.pdf. (April 2017). (Retrieved January 7, 2020).

[68] Bodo Winter. 2013. Linear models and linear mixed effects models in $\mathrm{R}$ with linguistic applications. http://arxiv.org/abs/1308.5499, CoRR abs/1308.5499 (2013).

[69] Thomas Wood and Ethan Porter. 2019. The Elusive Backfire Effect: Mass Attitudes' Steadfast Factual Adherence. Political Behavior 41, 1 (01 March 2019), 135-163. DOI : http://dx.doi.org/10.1007/s11109-018-9443-y

[70] YouTube. 2018. Building a better news experience on YouTube, together. https://youtube.googleblog.com/2018/ 07/building-better-news-experience-on.html. (July 2018). (Retrieved January 7, 2020). 